\title{
A Nearest Neighbor Search Algorithm for LR-LD on High SNR
}

\author{
Thae Thae Yu Khine, Daisuke Mitsunaga, Koji Araki and Hua-An Zhao \\ Department of Computer Science and Electrical Engineering, Kumamoto University, \\ 2-39-1 Kurokami, Chuo-ku, Kumamoto, 860-8555, Japan \\ E-mail: thaethaeyk@st.cs.kumamoto-u.ac.jp; cho@cs.kumamoto-u.ac.jp
}

\begin{abstract}
The faster decoding algorithm is still required in multiple-input multiple-output (MIMO) wireless communication technology. Lattice reduction (LR) technique can improve the performance of linear MIMO detection methods. LLL (Lenstra-Lenstra-Lovasz) algorithm is one of the LR techniques and it has being extensively used for better basis of channel matrix by removing noise. LR-aided detection algorithms involve two problems. The first problem is that the reduced basis is still non-orthogonal and most algorithms are devoted for finding a near orthogonal matrix. The second problem is that the feasible set of the detected symbol cannot be found without huge operation. In this paper, we propose a new algorithm which considers unknown transformed symbol to search the neighboring points on transformed basis of the channel matrix. This paper mainly focuses on the second problem to resolve the poor accuracy particularly in high signal-to-noise ratio (SNR). Our proposed algorithm can achieve better BER performance with low complexity and it is proven by simulations.
\end{abstract}

Keywords: multiple-input multiple-output (MIMO), LR-aided LDs, bit error rate (BER).

\section{Introduction}

Multiple-input multiple-output technology for wireless communication system has been extensively investigated in recent research with many antennas increasing from tens to hundreds due to its potential support for greater efficiency in data rate and higher reliability than a conventional technology like singleinput single-output (SISO) $)^{1,2}$. Zero-forcing (ZF), minimum-mean-square-error (MMSE) and maximum likelihood detection (MLD) are well known among the MIMO detection techniques. ZF and MMSE are linear detection techniques which provide low complexity with inferior performance in comparison with ML detection. ML detection can provide optimal performance when all transmitted codewords have equal probabilities. However, the ML detection still suffers from exponential complexity with the number of transmit antennas and the constellation size. Therefore, there has been challenging for low complexity and great performance of receiver techniques in large-scale MIMO systems.

Lattice reduction (LR) aided MIMO detection techniques are recently adopted in MIMO systems. Lattice reduction (LR) aided linear detection (LD) techniques can give the same BER performance as ML detection with low complexity. LR-aided linear detection algorithm attempts in finding a near orthogonal matrix like other algorithms ${ }^{3}$. The most widely used and well known LR-aided algorithm is LLL (Lenstra-Lenstra-Lovasz) algorithm. The LLL algorithm reduces the basis of channel matrix. The LR-aided detectors are based on the LLL algorithm which is originally used for reducing real lattice bases ${ }^{4}$. Lattice reduction is effective for interference between signals. When SNR is low, noise is larger than interference. When SNR is high, interference is larger than noise. LR can improve the performance by reducing interference on high SNR.

Among the studies on MIMO wireless communication technology in advance, an increase in 
the complexity is one of the MIMO problems in signal processing. In this paper, we propose a new algorithm focusing on the lattice reduction (LR) to improve the bit error rate (BER) performance. LR can significantly improve the BER performance in MIMO linear decoding using LLL (Lenstra-Lenstra-lovasz) algorithm. However, LLL algorithm has two problems $^{5,6}$. One is non-orthogonal reduced basis and another is finding the feasible set of detected symbol with round operation. A new proposed algorithm focuses on finding the feasible set of detected symbol with unknown transformed symbol and uses maximum likelihood method.

The remaining sections of this paper are organized as follows. Section 2 introduces the system model and some consideration on the decoding process. Section 3 introduces the lattice reduction (LR) technique and LLL ( Lenstra-Lenstra-lovasz) algorithm. Then the LR-aided linear detection (LD) based on zero-forcing (ZF) is described in Section 3 as well. Our proposed algorithm is introduced in Section 4. The simulation results and analysis are shown in Section 5. This paper is summarized in Section 6.

Notation: In this paper, real and imaginary parts of a complex number are denoted as $\mathfrak{R}($.$) and I (.) . The$ matrices are stated by uppercase letters like $A$ and vectors are stated by lowercase letters like $a$.

\section{System Model}

In MIMO wireless communication technology, multiple antennas at the transmitter simultaneously transmit a signal in order to achieve high speed. The receivers must extract the signal from the mixing as possible as the same to the transmitted signals. Therefore, signal decoding is complicate in MIMO wireless communication systems.

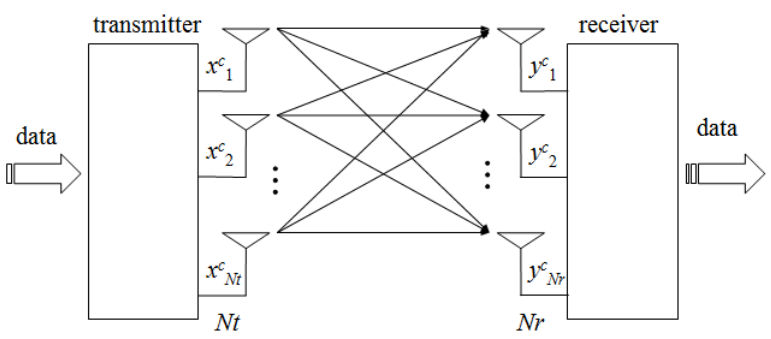

Fig. 1. MIMO system model.
In this paper, we consider a MIMO system as Fig. 1 with $N_{t}$ antennas for transmitting and $N_{r}$ antennas for receiving as

$$
y^{c}=H^{c} x^{c}+z^{c}
$$

where $y^{c} \in \mathbf{C}^{N r}$ is the received signal, $H^{c} \in \mathbf{C}^{N r \times N t}$ is a Rayleigh fading channel. $x^{c} \in \mathbf{C}^{N t}$ is the transmitted signal drawn from the M-Quadratic Amplitude Modulation (M-QAM) constellation set and $z^{c} \in \mathbf{C}^{N r}$ is the noise vector.

In LR, it is considered Eq. (1) is modified as follows.

$$
\begin{gathered}
y=H x+z \\
y=\left(\begin{array}{c}
\mathrm{R}\left(y^{c}\right) \\
\mathrm{I}\left(y^{c}\right)
\end{array}\right), z=\left(\begin{array}{c}
\mathrm{R}\left(z^{c}\right) \\
\mathrm{I}\left(z^{c}\right)
\end{array}\right), x=\left(\begin{array}{c}
\mathrm{R}\left(x^{c}\right) \\
\mathrm{I}\left(x^{c}\right)
\end{array}\right) \\
H=\left(\begin{array}{cc}
\mathrm{R}\left(H^{c}\right) & -\mathrm{I}\left(H^{c}\right) \\
\mathrm{I}\left(H^{c}\right) & \mathrm{R}\left(H^{c}\right)
\end{array}\right) .
\end{gathered}
$$

Then $y \in \mathbf{R}^{K}, z \in \mathbf{R}^{K}, x \in \mathbf{R}^{N}, H \in \mathbf{R}^{K \times N}$, where $\mathrm{K}$ $=2^{N r}, \mathrm{~N}=2^{N t}$.

\subsection{MIMO linear detection}

The zero-forcing (ZF) linear detection suppresses all the mutual interference between the layers, which can be accomplished by the Moore-Penrose pseudo-inverse of the channel matrix. The transmit signal can be recovered as

$$
\hat{s}_{Z F}=Q\left(H^{\dagger} y\right),
$$

where $H^{\dagger}=\left(H^{H} H\right)^{-1} H^{H}$, and $Q($.$) is the symbol-wise$ quantizer to the constellation $\mathrm{S}$. When it comes to taking noise amplification into consideration, the minimal-mean-square-error (MMSE) linear detection is proposed. The transmit signal can be concisely approached by

$$
\hat{s}_{M M S E}=Q\left(\underline{H}^{\dagger} \underline{y}\right),
$$

where $\underline{H}=\left[\begin{array}{l}H \\ \sigma I_{N}\end{array}\right], \underline{y}=\left[\begin{array}{l}y \\ 0\end{array}\right]$ are the augment channel matrix and the extended receive vector, respectively. Also, $\sigma$ is the noise power and $\mathrm{I}_{N}$ is the $N_{r} \times N_{r}$ unitary matrix. 


\section{LR-LD Algorithm}

Lattice reduction (LR) technique can obtain a near orthogonal matrix which improves the BER performance. LLL algorithm is a typical algorithm of LR techniques to find the unimodular transformation $\bar{H}=H T$, such as $\bar{H}$ is the reducing basis of channel matrix $H$ and near orthogonal matrix, $T$ is unimodular matrix and $H$ is near orthogonal matrix. LLL algorithm finds the shortest and nearest orthogonal basis vector from input vectors using the Gram-Schmidt orthonormalization. The LR-aided LDs in zero-forcing (ZF) case based on the reduced basis $H$ are performed as follows ${ }^{5}$. Note

$$
y=H T T^{-1} x+z=\bar{H} \bar{x}+z
$$

where $\bar{H}=H T$ and $\bar{x}=T^{-1} x$. By applying scaling and shifting on $\bar{x}$, i.e., $\left(x-1_{N \times 1}\right) / 2$ where $1_{N \times 1} \in \mathrm{R}_{N \times 1}$ is the matrix with entries 1 , we transfer the constellation set $S$ to consecutive integer sets, which are further transferred to the lattice-reduction domain as $b=T^{-1}(x$ $-1_{N \times 1}$ ) / 2, whose elements are in the consecutive integer sets as well. Then we know, $\bar{x}=T^{-1} x=2 b+T^{-1} 1_{N \times 1}$, Eq. (5) is equivalent to

$$
y=\bar{H} \bar{x}+z=2 \bar{H} b+\bar{H} T^{-1} 1_{N \times 1}+z
$$

We obtain the estimation of $b$ as

$$
\hat{b}=\left\lfloor\frac{1}{2} \bar{H}^{\dagger}\left(y-\bar{H} T^{-1} 1_{N \times 1}\right)\right\rceil
$$

where [.] is the rounding function and ()$\dagger$ is the pseudoinverse of ( ). Then the estimation $x$ is

$$
\hat{\bar{x}}=2 \hat{b}+T^{-1} 1_{N \times 1} \text {. }
$$

\subsection{Principle of LLL reduction}

Before we apply LLL reduction for channel matrix, we need to transform QR decomposition for channel matrix, which is obtained by using Gram-Schmidt orthonormalization as follow

$$
H=\bar{Q} \bar{R},
$$

where $\bar{Q} \in \mathrm{R}^{K \times N}$ and $\bar{R} \in \mathrm{R}^{N \times N}$ is the unitary matrix and the upper triangle matrix, respectively. These matrix show that the smaller the value of $\left|\bar{R}_{j, k}\right|$ (i.e., the bigger the value of $\left.\left|\bar{R}_{k, k}\right|\right)$ is, the more orthogonal the basis of the channel matrix is

In order to obtain more orthogonal basis of channel matrix, we transform the upper triangle matrix $\bar{R}$ satisfying with the two conditions as follow.

$$
\begin{gathered}
\left|\bar{R}_{k \times l}\right| \leq \frac{1}{2}\left|\bar{R}_{l \times l}\right|,(0 \leq l<k \leq \mathrm{N}), \\
\delta\left|\bar{R}_{k-1, k-1}\right|^{2} \leq\left|\bar{R}_{k \times k}\right|^{2}+\left|\bar{R}_{k-1, k}\right|^{2}, \\
(k=2, \ldots, N) ;
\end{gathered}
$$

where $\delta$ is a parameter to determine how rate the diagonal elements reduce and it is commonly defined as

$$
\frac{1}{4}<\delta \leq 1
$$

These conditions are used to transform upper triangle matrix $\bar{R}$ maximize its diagonal elements. When the diagonal elements of the upper triangle matrix $\bar{R}$ is dissatisfying the condition in Eq. (11), i.e.,

$$
\delta\left|\bar{R}_{k-1, k-1}\right|^{2}>\left|\bar{R}_{k, k}\right|^{2}+\left|\bar{R}_{k-1, k}\right|^{2}
$$

We calculate the given rotation matrix defined as

$$
\begin{aligned}
& G=\left[\begin{array}{cc}
\alpha & \beta \\
-\beta & \alpha
\end{array}\right], \\
& \alpha=\frac{\mid \bar{R}_{k-1, k-1 \mid}}{\sqrt{\left|\bar{R}_{k-1, k-1}\right|^{2}+\left|\bar{R}_{k, k-1}\right|^{2}}}, \beta=\alpha \frac{\bar{R}_{k, k-1}}{\bar{R}_{k-1, k-1}},
\end{aligned}
$$

and we update the unitary matrix $\bar{Q}$ and the upper triangle matrix $\bar{R}$ multiplying the givens rotation matrix $G$ as follow,

$$
\begin{aligned}
& \bar{R}_{k-1: k, k-1: N}=G \bar{R}_{k-1: k, k-1: N,}, \\
& \bar{Q}_{:, k-1: k}=\bar{Q}_{:, k-1: k} G^{H}
\end{aligned}
$$

The LLL algorithm repeats these calculations until satisfying with the condition of (11). 


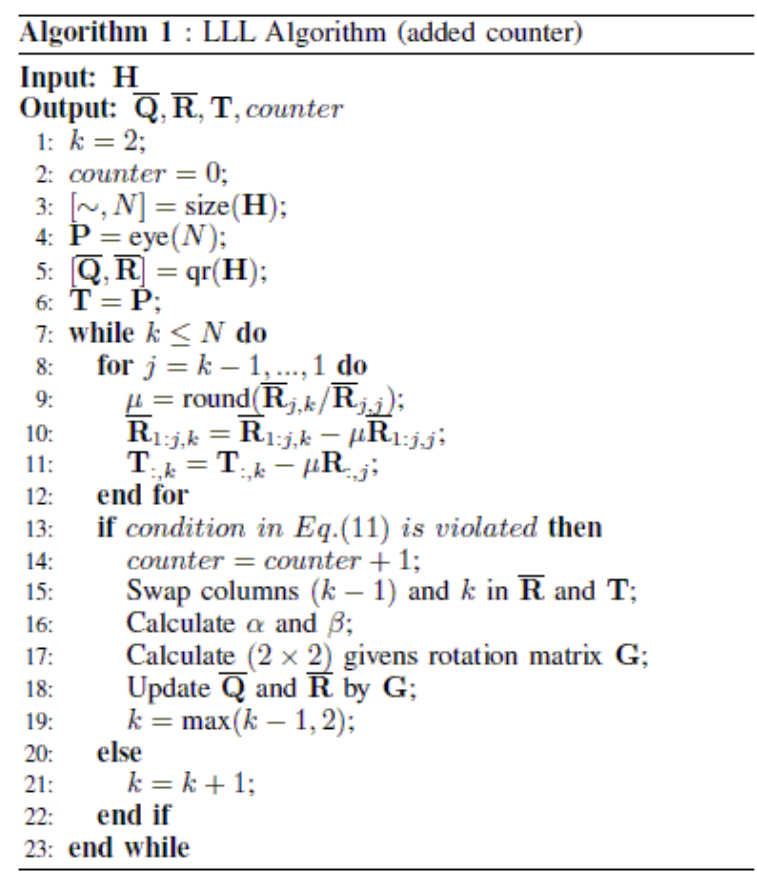

Fig. 2. LLL algorithm.

\section{Proposed Algorithm}

Estimation of $b$ using Eq. (7) is not optimal because it has two reasons ${ }^{3}$, which are:

- $\quad H$ is not precise orthogonal.

- regarding the consecutive integer set as the feasible set of $\boldsymbol{b}$ is not exact.

Lattice reduction can suffer SNR loss due to these two reasons ${ }^{2}$. Improving these two reasons can make better result. Many paper focus to improve the first reason. In this paper, we propose an algorithm which focuses to improve the second reason.

Note the maximum likelyhood detection of $\boldsymbol{b}$ is

$$
\hat{b}=\arg \min \|\bar{y}-2 \bar{H} b\|^{2},
$$

where $\bar{y}=y-2 \bar{H} T^{-1} 1_{N \times 1}$ and $\mathrm{S}^{\prime}$ is a feasible set of $b$.

In previous LR-aided LD algorithms apply simple rounding operation such as Eq. (7). However, it is not exact for the feasible set of $b$ which is integer vectors in a polyhedron. We consider an antenna and 16-QAM modulation and suppose that unimodular matrix

$$
T=\left|\begin{array}{ll}
1 & 1 \\
0 & 1
\end{array}\right|
$$

is created and then the constellation of $\mathrm{x}$ and the feasible set of $b$ are shown in Fig. 3 and Fig. 4, respectively.

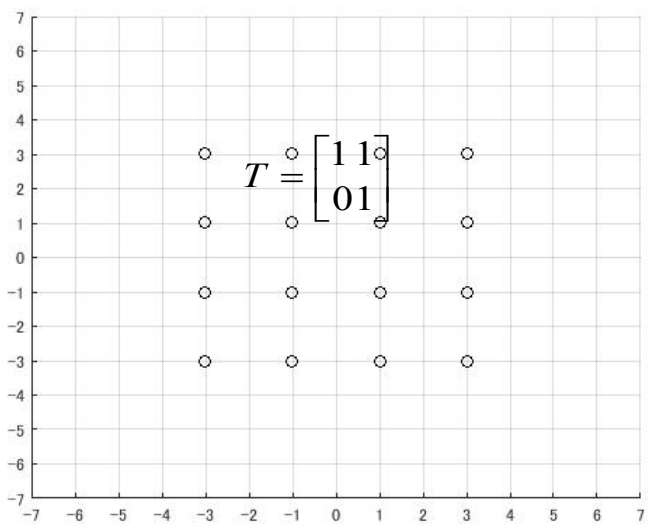

Fig. 3. Constellation of $\mathrm{x}$.

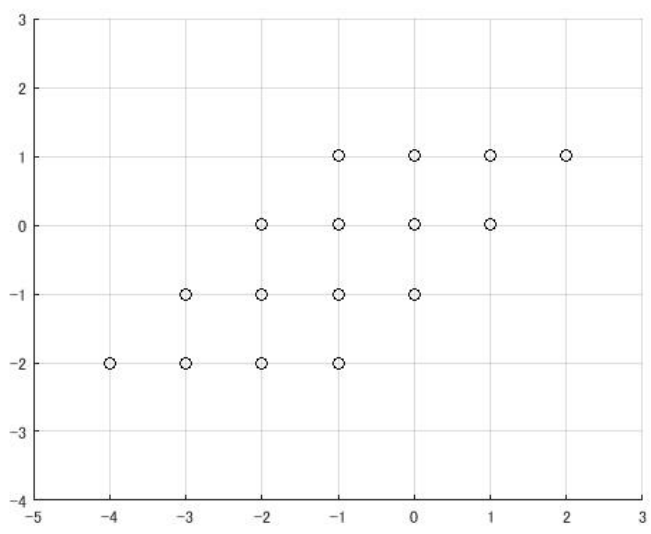

Fig. 4. Feasible set of $b$.

In this case, finding all transformed coordinates of symbol sets in the space as shown in Fig. 4 is inefficient. Also finding the Euclidean distance between the received signal and the floating point symbols such as the Eq. (17) are significantly degrade the high-speed performance of the LR-LD. A new proposed algorithm improves the BER performance of LR-aided LD without symbol coordinates on the transformed space and floating point operation.

First, we find the $\hat{b}$ using Eq. (7). Second, an integer coordinate set $a$ that surrounds the received signal is created based on relation between $\hat{\boldsymbol{b}}$ and $\boldsymbol{b}$, as shown in Fig. 5. Finally, we find the transmitted signal by searching the integer coordinates set within $\boldsymbol{a}$ if defined as follows. 


$$
\bar{a}=2 a+T^{-1} 1_{N \times 1},
$$

then $\bar{a}$ is transformed into distorted integer coordinates set as shown in Fig. 6 . When you search a range that has been surrounded by the integer coordinates set $a, \hat{b}$ is

$$
\begin{aligned}
& \qquad \hat{b}=\arg \min \|\bar{a}-H x\|^{2}, \\
& \text { s.t. } \bar{a}, b \in \mathrm{S}^{\prime}
\end{aligned}
$$

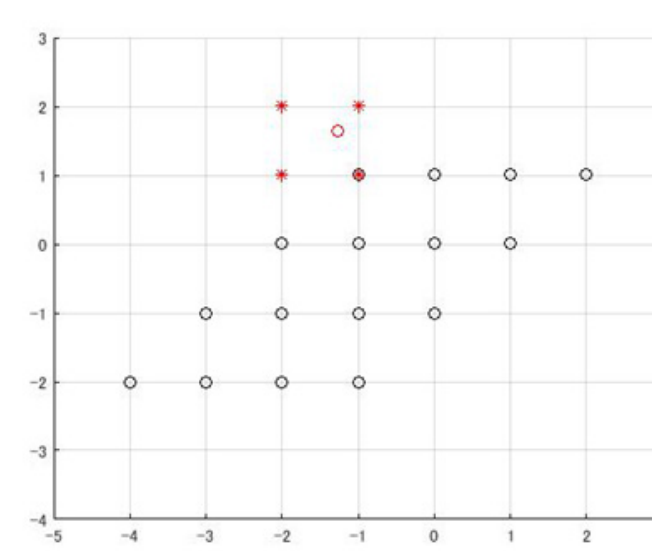

Fig. 5. Received signal (red circle mark) and search integer coordinate set a (red asterisk).

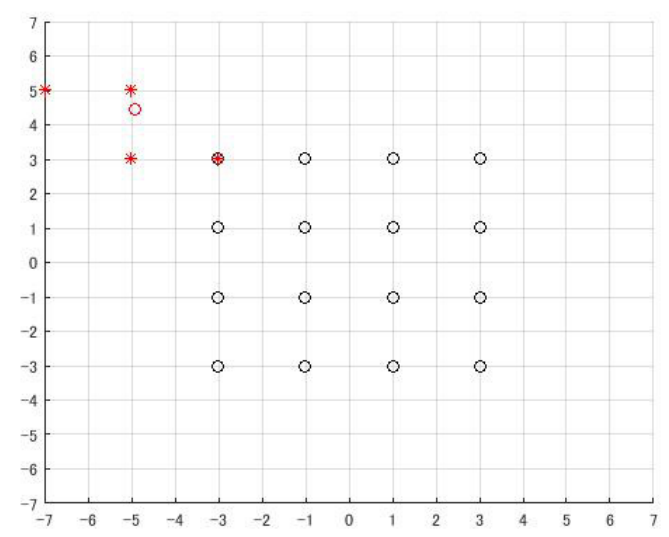

Fig. 6. Received signal (red circle mark) and search integer coordinate set a (red asterisk).

In a typical QAM modulation, it is sufficient to look for coordinates of symbols that match the search integer coordinates set $a$, because all the symbol set are integer vectors. Therefore, it is not necessary to calculate the Euclidean distance. This proposed algorithm shown as Fig. 7 allows the decoding when the received signal is slightly away from the constellation. In other words, decodable range can be expanded in accordance with the search range.

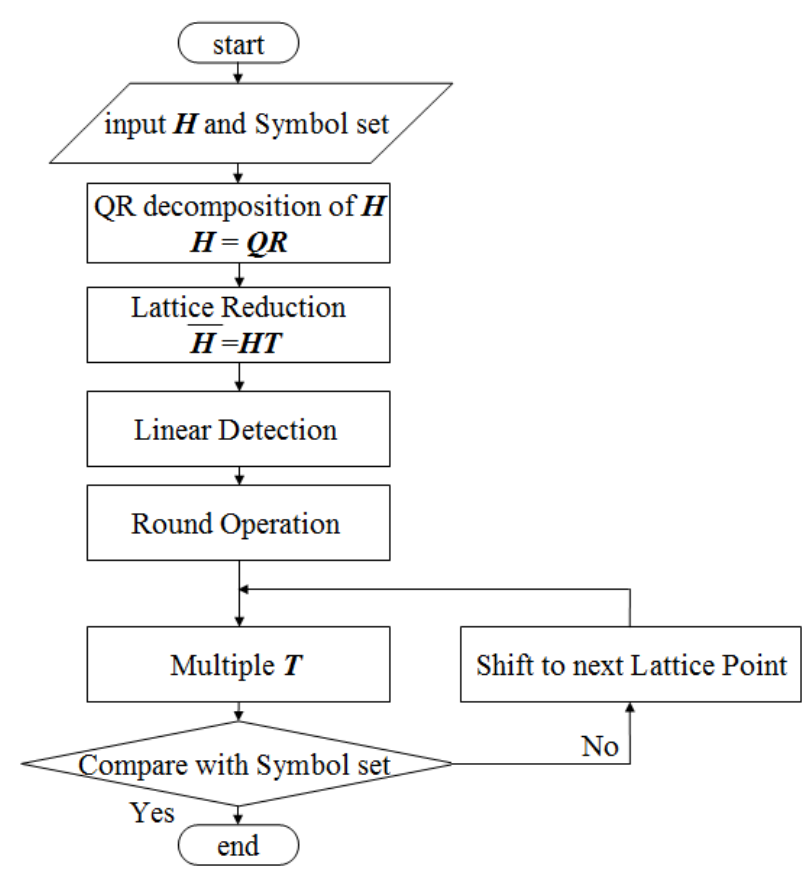

Fig. 7. Flowchart of proposed algorithm.

\section{Simulation Results and Analysis}

The performance of the proposed algorithm is proven by computer simulation over various MIMO systems. In this paper, we compare the BER performance of $\mathrm{ZF}$, LR-ZF, proposed LR-ZF, LR-MMSE and proposed LRMMSE.

\subsection{BER performance}

Fig. 8 (a), 8(c) and $8(\mathrm{e})$ show respectively the BER performance of $4 \times 4,8 \times 8,16 \times 16 \mathrm{MIMO}$ systems in 4-QAM modulation. Fig. 8(b), 8(d) and $8(\mathrm{f})$ show respectively the BER performance of $4 \times 4,8 \times 8,16 \times 16$ MIMO systems in 16-QAM modulation.

According to the results shown in Fig. 8(a), 8(b), $8(\mathrm{c})$ and $8(\mathrm{~d})$, it can be seen that the proposed algorithm for each LD has better BER performance than previous LR-LD algorithms. From the view of modulation schemes, 16-QAM has smaller performance gain than 4QAM because 16-QAM has smaller expansion rate than 4-QAM where expansion rate is the ratio of an enlarged decodable range. However, the performance gains of 4QAM and 16-QAM are slightly better than the previous LR-LD algorithms for higher order of antenna as shown in Fig. 8(e) and Fig. 8(f). 


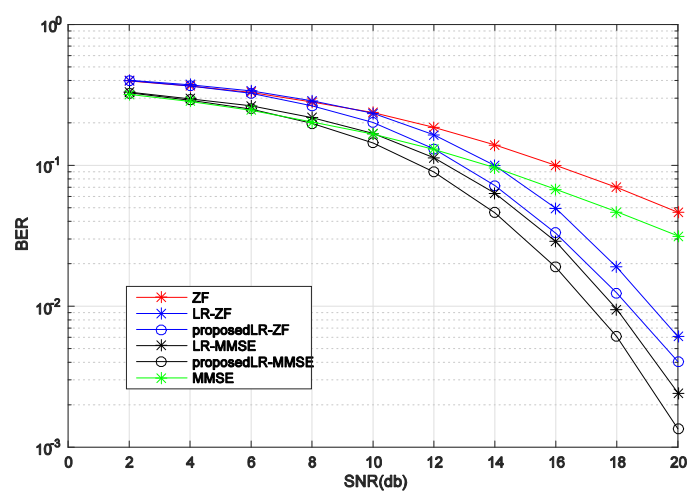

(a) 4 x 4 MIMO, 4-QAM.

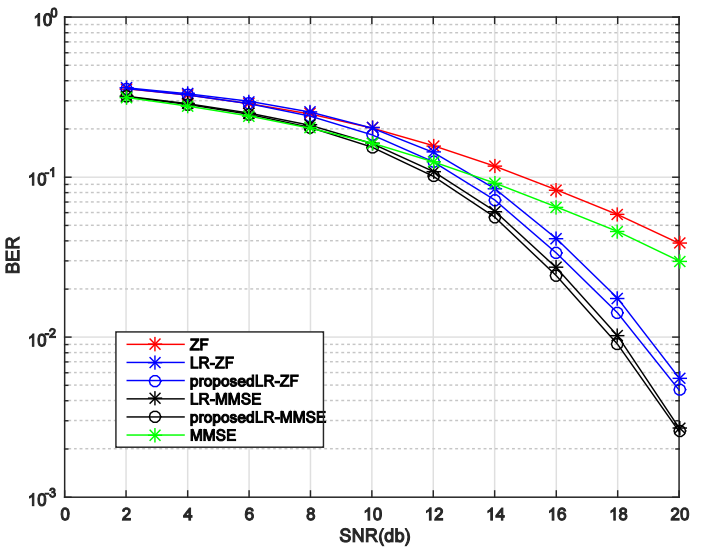

(b) 4 x 4 MIMO, 16-QAM.

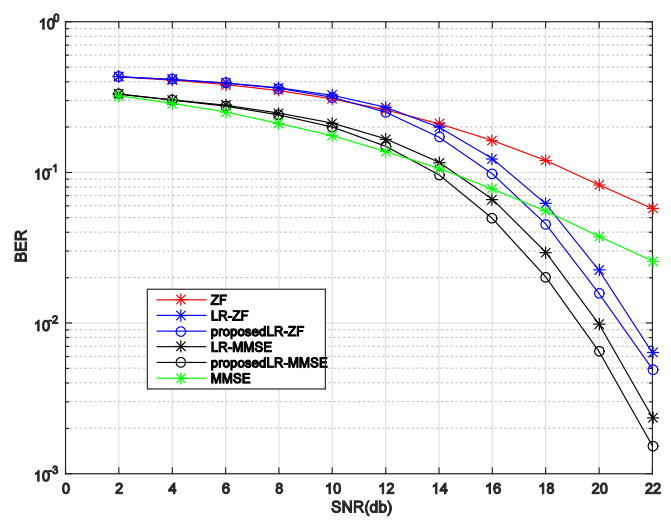

(c) 8 x 8 MIMO, 4-QAM.

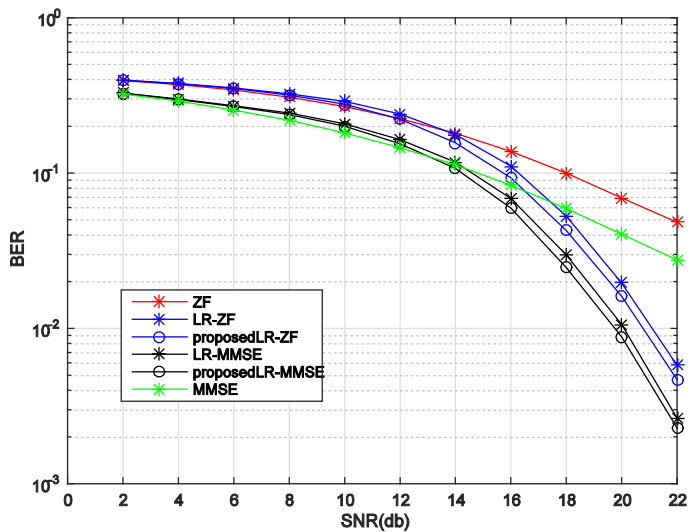

(d) 8 × 8 MIMO, 16-QAM.

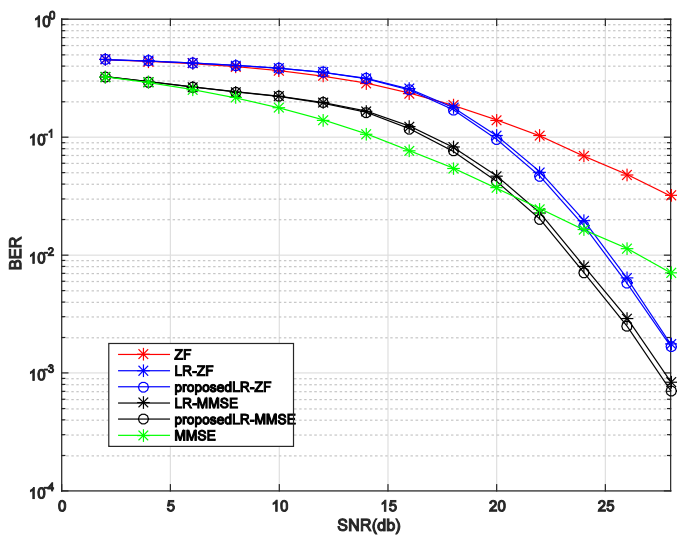

(e) 16 x 16 MIMO, 4-QAM.

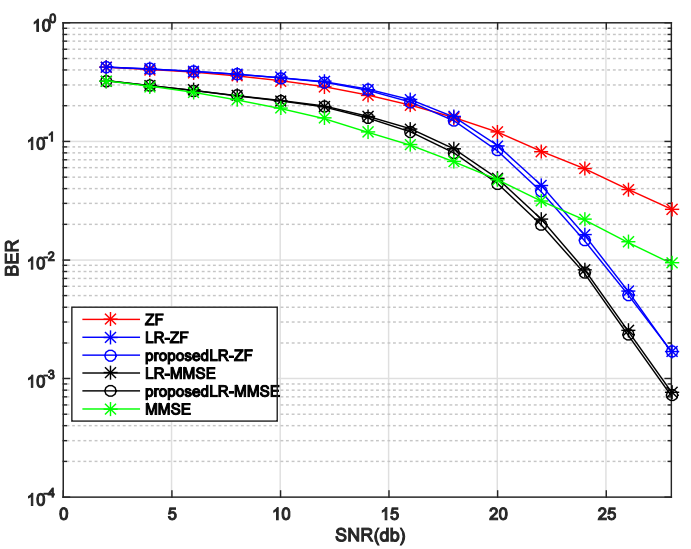

(f) $16 \times 16$ MIMO, 16-QAM.

Fig.8. BER performances with 4x4, 8x8, 16x16 MIMO and 4, 16-QAM.Flowchart of proposed algorithm. 


\subsection{Complexity analysis}

Our proposed algorithm mainly includes two parts. The first part is the derivation of a unimodular matrix which can obtain a near orthogonal matrix. In this part, our algorithm is the same as the previous LR-LD algorithm. In second part, the proposed algorithm extends the received signal point calculated in LD to neighbourhood point. In this part, our algorithm is more complex than previous LR-LD algorithm because the number of calculation for returning to original basis increases depending on the number of points. However, calculation order is $O\left(n^{2}\right)$ since it is a simple operation which only calculates the product and sum of a simple matrix with integer values. Calculation order of QR decomposition to simplify the decoding in the MIMO system is $O\left(n^{3}\right)$. Therefore, it can be said the increase in the complexity of the proposed algorithm are minimal.

\section{Conclusion}

The detection in MIMO system is important as many antennas are used to transmit and receive signals to imp rove the performance. Lattice reduction (LR) can achieve the better performance by reducing the interferrence between signals on high SNR. The decoding algorithm of MIMO system with its low complexity and great performance is developed from the LR based algorithm. Two problems of LR are that the reduced basis is still non-orthogonal and the feasible set of the detected symbol cannot be found without huge opera- tion. In this paper, we emphasize on the second problem. We propose to assume the unknown symbol set on new basis by LR and we expanded decodable range by only simple integer operations for high BER performance. We demonstrated the computer simulatio ns from lower to higher order of antenna and modulation schemes. Therefore it can be said that our algorithm improves the BER performance of LR-LD on high SNR.

\section{Acknowledgements}

This work is supported by Japan Society for the Promotion of Science under Grant No.26420362.

\section{References}

1. CHEN, Zhiyong; DAI, XUxhu. A Fast complex lattice reduction algorithm for SIC-based MIMO detection. In: Communicaiton (ICC), 2015 IEEE International Conference on. IEEE, 2015. P. 2283-2288

2. SINGHAI, Kamal A.; DATTA, Tanmoy; CHOCKALINGAM, Ananthanarayanan, Lattice reduction aided detection in large-MIMO systems. In: Signal Processing Advances in Wireless Communications (SPAWC), 2013 IEEE $14^{\text {th }}$ Workshop on. IEEE, 2013. P. 594-598.

3. Yunchao Dong, Chen Liu, Feng Lu, and Hua-An ZHAO "An Improved Detection Algorithm Based on Lattice Reduction for MIMO System" Department of Computer Science and Electrical Engineering.

4. GAN, ying Hung: MOW, Wai Ho, Complex lattice reduction algorithms for low-complexity MIMO detection. In: Global Telecommunicaitons Conference, 2005. GLOBECOMf05. IEEE, IEEE, 2005. p. 5 pp-2957.

5. H. Yao, and G.W. Wornell, "Lattice-reduction-aided detectors for MIMO communication systems" Proc.IEEE Global Tele. Conf., Nov. 2002, pp. 424-428.

6. Yuta Kawaguchi, Satoshi Denno, Hidekazu Murata and Daisuke Umehara, "Lattice reduction aided SIC Detector and its Performance," IEICE Tech. Rep, col.115, no 160, RCS2015-218, pp. 163-167, July 2015. 